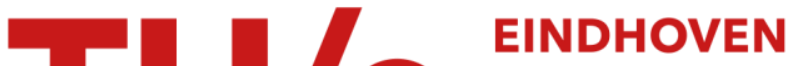 \\ UNIVERSITY OF \\ TECHNOLOGY
}

\section{Stability of negative ionization fronts: Regularization by electric screening?}

Citation for published version (APA):

Arrayas, M., \& Ebert, U. (2004). Stability of negative ionization fronts: Regularization by electric screening? Physical Review E - Statistical, Nonlinear, and Soft Matter Physics, 69(3), 036214-1/10. [036214].

https://doi.org/10.1103/PhysRevE.69.036214

DOI:

10.1103/PhysRevE.69.036214

Document status and date:

Published: 01/01/2004

\section{Document Version:}

Publisher's PDF, also known as Version of Record (includes final page, issue and volume numbers)

\section{Please check the document version of this publication:}

- A submitted manuscript is the version of the article upon submission and before peer-review. There can be important differences between the submitted version and the official published version of record. People interested in the research are advised to contact the author for the final version of the publication, or visit the $\mathrm{DOI}$ to the publisher's website.

- The final author version and the galley proof are versions of the publication after peer review.

- The final published version features the final layout of the paper including the volume, issue and page numbers.

Link to publication

\section{General rights}

Copyright and moral rights for the publications made accessible in the public portal are retained by the authors and/or other copyright owners and it is a condition of accessing publications that users recognise and abide by the legal requirements associated with these rights.

- Users may download and print one copy of any publication from the public portal for the purpose of private study or research.

- You may not further distribute the material or use it for any profit-making activity or commercial gain

- You may freely distribute the URL identifying the publication in the public portal.

If the publication is distributed under the terms of Article 25fa of the Dutch Copyright Act, indicated by the "Taverne" license above, please follow below link for the End User Agreement:

www.tue.nl/taverne

Take down policy

If you believe that this document breaches copyright please contact us at:

openaccess@tue.nl

providing details and we will investigate your claim. 


\title{
Stability of negative ionization fronts: Regularization by electric screening?
}

\author{
Manuel Arrayás ${ }^{1,2}$ and Ute Ebert ${ }^{2,3}$ \\ ${ }^{1}$ Universidad Rey Juan Carlos, Departmento de Física, Tulipán s/n, 28933, Móstoles, Madrid, Spain \\ ${ }^{2}$ Centrum voor Wiskunde en Informatica (CWI), P.O. Box 94079, 1090 GB Amsterdam, The Netherlands \\ ${ }^{3}$ Department of Physics, Eindhoven University of Technology, The Netherlands
}

(Received 24 July 2003; published 31 March 2004)

\begin{abstract}
We recently have proposed that a reduced interfacial model for streamer propagation is able to explain spontaneous branching. Such models require regularization. In the present paper we investigate how transversal Fourier modes of a planar ionization front are regularized by the electric screening length. For a fixed value of the electric field ahead of the front we calculate the dispersion relation numerically. These results guide the derivation of analytical asymptotes for arbitrary fields: for small wave-vector $k$, the growth rate $s(k)$ grows linearly with $k$, for large $k$, it saturates at some positive plateau value. We give a physical interpretation of these results.
\end{abstract}

DOI: 10.1103/PhysRevE.69.036214

PACS number(s): 47.54.+r, 52.80.-s, 52.35. $-\mathrm{g}, 05.45 .-\mathrm{a}$

\section{INTRODUCTION}

Streamers generically appear in electric breakdown when a sufficiently high voltage is suddenly applied to a medium with low or vanishing conductivity. They consist of extending fingers of ionized matter and are ubiquitous in nature and technology. Frequently they are observed to branch $[1,2]$. There is a traditional qualitative concept for streamer branching based on rare photoionization events [3-7]. However, our recent work $[8-10]$ has shown that even the simplest, fully deterministic streamer model without photoionization can exhibit branching. In particular, we have proposed [8] that a streamer approaching the Lozansky-Firsov limit of ideal conductivity [11] can branch spontaneously due to a Laplacian interfacial instability [12]. This mechanism is quite different from the one proposed previously. It requires less microscopic physical interaction mechanisms, but is based on internal structure of the propagating streamer head with a thin space charge layer. Analytical branching predictions from the simplest type of interfacial approximation can be found in Ref. [10].

However, the simple interfacial model investigated in Ref. [10] requires regularization to prevent the formation of cusps. The nature of this regularization has to be derived from the underlying gas discharge physics; it recently has been a subject of debate $[13,14]$. We argue that one regularization mechanism is generically inherent in any discharge model, namely the thickness of the electric screening layer. This is the subject of the present paper: we study how the electric screening layer present in the partial differential equations of the electric discharge influences the stability of an ionization front, correcting the simple interfacial model proposed in Refs. $[8,11,12,15]$ and solved in Ref. [10]. To be precise, we derive the dispersion relation for transversal Fourier modes of a planar ionization front. We treat a negative front in a model as in $[8,9,12,15-17]$, but with vanishing electron diffusion and under the assumption that the state ahead of the ionization front is completely nonionized. We have shown previously that the analysis of the full model $[8,9,12,15-17]$ is mathematically nonstandard and challenging due to the "pulled" nature $[18,19]$ of the front. Pulling is a mode of front propagation where the spatially half-infinite leading edge of a front dominates its behavior. However, for vanishing electron diffusion and propagation into a nonionized state, the leading edge of the ionization front is completely eliminated and replaced by a discontinuous jump of the electron density to some finite value. This corresponds to the fact that neglecting electron diffusion changes the equation of electron motion from parabolic to hyperbolic type. Putting $D_{e}=0$ in the present paper and considering propagation into a nonionized state, we get rid of leading edge and pulling, but in turn we have to analyze discontinuous fronts.

Here we anticipate the result of the paper: if the field far ahead of a planar negative ionization front is $E_{\infty}$, then a transversal Fourier perturbation with wave vector $k$ grows with rate

$$
s(k)= \begin{cases}\left|E_{\infty}\right| k & \text { for } k \ll \alpha\left(E_{\infty}\right) / 2, \\ \left|E_{\infty}\right| \alpha\left(E_{\infty}\right) / 2 & \text { for } k \gg \alpha\left(E_{\infty}\right) / 2,\end{cases}
$$

where $\alpha(E)$ is the effective impact ionization coefficient within a local field $E$. The parameter $\alpha$ sets the size of the inverse electric screening length. The behavior for large $k$ is a correction to the interfacial model treated in Ref. [10]; in that model we would have $s(k)=\left|E_{\infty}\right| k$ for all $k$. The asymptotes (1) have been quoted already in Refs. $[8,15]$, however, without derivation. Their derivation based on numerical results and asymptotic analysis together with a discussion of the underlying physical mechanisms are the content of the present paper.

In detail, the paper is organized as follows. In Sec. II we summarize the minimal streamer model in the limit of vanishing diffusion and recall multiplicity, selection, and analytical form of uniformly translating planar front solutions; we then derive the asymptotic behavior at the position of the shock and far behind the shock, and we discuss two degeneracies of the problem. In Sec. III we set up the framework of the linear perturbation analysis for transversal Fourier modes, first the equation of motion and then the boundary conditions and the solution strategy. In Sec. IV we present numerical results for the dispersion relation for field $E_{\infty}$ $=-1$, and we derive the asymptotes (1) analytically for ar- 
bitrary $E_{\infty}$. The small $k$ limit is related to one of the degeneracies of the unperturbed problem, for the large $k$ limit we also present a physical interpretation. Section V contains conclusions and outlook.

\section{MINIMAL STREAMER MODEL AND PLANAR FRONT SOLUTIONS}

\section{A. The minimal model}

We investigate the minimal streamer model, i.e., a "fluid approximation" with local field-dependent impact ionization reaction in a nonattaching gas such as argon or nitrogen $[8,9,12,15-17,20]$. For physical parameters and dimensional analysis, we refer to our previous discussions in Refs. $[8,9,12,15]$. When electron diffusion is neglected $\left(D_{e}=0\right)$, the dimensionless model has the form

$$
\begin{gathered}
\partial_{t} \sigma-\nabla \cdot(\sigma \mathbf{E})=\sigma f(\mathbf{E}), \\
\partial_{t} \rho=\sigma f(\mathbf{E}), \\
\nabla \cdot \mathbf{E}=\rho-\sigma, \quad \mathbf{E}=-\nabla \phi,
\end{gathered}
$$

where $\sigma$ is the electron density, $\rho$ is the ion density, and $\mathbf{E}$ is the electric field. Here the electron current is assumed to be $\sigma \mathbf{E}$ and the ion current is neglected. Electron-ion pairs are assumed to be generated with rate $\sigma f(\mathbf{E})=\sigma|\mathbf{E}| \alpha(|\mathbf{E}|)$, where $\sigma|\mathbf{E}|$ is the absolute value of electron current and $\alpha(\mathbf{E})$ the effective impact ionization cross section within a field $\mathbf{E}$. Hence $f(\mathbf{E})$ is

$$
f(\mathbf{E})=|\mathbf{E}| \alpha(|\mathbf{E}|) .
$$

For numerical calculations, we use the Townsend approximation

$$
\alpha(|\mathbf{E}|)=e^{-1 /|\mathbf{E}|}
$$

For analytical calculations, an arbitrary function $\alpha(\mathbf{E})$ can be chosen where we only assume that

$$
f(\mathbf{E})=f(|\mathbf{E}|) \quad \text { and } \quad \alpha(0)=0 .
$$

The last identity entails that $f(0)=0=f^{\prime}(0)$. For certain results we also need that $\alpha(|\mathbf{E}|)$ does not decrease when $|\mathbf{E}|$ increases, hence that $\alpha^{\prime} \geqslant 0$.

Note that the electrons are the only mobile species and the source of additional ionization, while ion density $\rho$ and electric potential $\phi$ or field $\mathbf{E}$ follow the dynamics of the electron density $\sigma$, and couple back onto it.

\section{B. Uniformly translating ionization fronts: Analytical solutions and multiplicity}

We now recall essential properties of uniformly translating planar front solutions of Eqs. (2)-(5) and (7). First of all, a constant mode of propagation requires a planar density distribution that we assume to vary only in the $z$ direction: $(\sigma, \rho)=[\sigma(z, t), \rho(z, t)]$, the particle densities for large positive $z$ are assumed to vanish. The field far ahead of the front in the nonionized region at $z \rightarrow \infty$ has to be constant in time and as a consequence of Eq. (4) also constant in space,

$$
\mathbf{E}= \begin{cases}E_{\infty} \hat{z}, & z \rightarrow+\infty, \\ 0, & z \rightarrow-\infty,\end{cases}
$$

where $\hat{z}$ is the unit vector in $z$ direction. For the boundary condition at $z \rightarrow-\infty$ we assumed that the ionized region behind the front extends to $-\infty$. This implies that a fixed amount of charge $\int(\rho-\sigma) d z=E_{\infty}$ is traveling within the front according to Eqs. (4) and (8), and no currents flow far behind the front in the ionized and electrically screened region.

For the further analysis, a coordinate system $(x, y, \xi=z$ $-v t$ ) moving with velocity $v$ in the $z$ direction is used. Then Eqs. (2)-(4) read

$$
\begin{gathered}
\partial_{t} \sigma-v \partial_{\xi} \sigma-(\rho-\sigma) \sigma+(\nabla \sigma) \cdot(\nabla \phi)-\sigma f(|\nabla \phi|)=0, \\
\partial_{t} \rho-v \partial_{\xi} \rho-\sigma f(|\nabla \phi|)=0 \\
\rho-\sigma+\nabla^{2} \phi=0
\end{gathered}
$$

where we expressed all quantities by electron density $\sigma$, ion density $\rho$, and electric potential $\phi$.

A front propagating uniformly with velocity $v$ is a solution of Eqs. (8) and (9), where $\sigma, \rho$, and $\phi$ depend on $\xi$ only. With $\nabla \phi=\partial_{\xi} \phi \hat{z}=-E \hat{z}$, such a front solves

$$
\begin{gathered}
(v+E) \partial_{\xi} \sigma+(\rho-\sigma) \sigma+\sigma f(|E|)=0, \\
v \partial_{\xi} \rho+\sigma f(|E|)=0, \\
\rho-\sigma-\partial_{\xi} E=0 .
\end{gathered}
$$

For use in the later sections, we now briefly recall the analytical solutions [12] of these equations. Subtract Eq. (11) from Eq. (10), use Eq. (12) to get a complete differential, integrate and use Eq. (12) again to get $-v \partial_{\xi} E+\sigma E$ $=$ const. The integration constant is fixed by the condition $E \rightarrow 0$ at $\xi \rightarrow-\infty$ from Eq. (8), and we find

$$
-v \partial_{\xi} E+\sigma E=0
$$

The front equations then reduce to two ordinary differential equations for $\sigma$ and $E$,

$$
\begin{gathered}
\partial_{\xi}[(v+E) \sigma]=-\sigma f(E), \quad f(E)=|E| \alpha(E), \\
v \partial_{\xi} \ln |E|=\sigma,
\end{gathered}
$$

which can be solved analytically as

$$
\begin{gathered}
\sigma[E]=\frac{v}{v+E} \rho[E], \\
\rho[E]=\int_{|E|}^{\left|E_{\infty}\right| f(x)} \frac{d x}{x} d x=\int_{|E|}^{\left|E_{\infty}\right|} \alpha(x) d x,
\end{gathered}
$$




$$
\xi_{2}-\xi_{1}=\int_{E\left(\xi_{1}\right)}^{E\left(\xi_{2}\right)} \frac{v+x}{\rho[x]} \frac{d x}{x}
$$

This gives us $\sigma$ and $\rho$ as functions of $E$, and the space dependence $E=E(\xi)$ implicitly as $\xi=\xi(E)$ in the last equation. It follows immediately from Eq. (17) that $E(\xi)$ is a monotonic function, and hence that the space charge $q=\rho$ $-\sigma=\partial_{\xi} E$ has the same sign everywhere. According to Eq. (16), $\rho(\xi)$ is a monotonic function, too.

Up to now, the front velocity $v$ as a function of the asymptotic field $E_{\infty}$ is not yet fixed. Indeed for any nonvanishing far field $E_{\infty}$, there is a continuous family of uniformly translating front solutions parametrized by $v[12,21]$, since the front propagates into an unstable state [18]. In particular, for $E_{\infty}>0$ there is a dynamically stable solution for any velocity $v \geqslant 0$, and for $E_{\infty}<0$, there is a dynamically stable solution for any $v \geqslant\left|E_{\infty}\right|$. These bounds on $v$ can be derived directly from Eqs. (15)-(17) with boundary condition (8) and the condition that the densities $\sigma$ and $\rho$ are non-negative for all $\xi$.

This continuous family of solutions parametrized by $v$ is associated with an exponentially decaying electron density profile in the leading edge $[12,18]$ : an electron profile that asymptotically for large $\xi$ decays like $\sigma(\xi) \propto e^{-\lambda \xi}$ with $\lambda \geqslant 0$, will propagate with velocity

$$
v=-E_{\infty}+\frac{f\left(E_{\infty}\right)}{\lambda} \text { in a field } E_{\infty}<0 .
$$

It will "pull" an ionization front along with the same speed. [For $E_{\infty}>0$, the same equation applies for all $\lambda$ $\geqslant f\left(E_{\infty}\right) / E_{\infty}$, hence for $v \geqslant 0$.]

\section{Dynamical selection of the shock front solution and its particular properties}

In practice, not all these uniformly propagating solutions are observed as asymptotic solutions of the full dynamical problem Eqs. (2)-(4), but only a specific one that is called the selected front. For a negative ionization front, it propagates with the velocity [12]

$$
v=\left|E_{\infty}\right| \text { for } E_{\infty}<0 .
$$

The selection takes place through the initial conditions [18]: If the electron density strictly vanishes beyond a certain point $\xi_{0}$ at time $t=0$

$$
\sigma=0=\rho \text { for } \xi>\xi_{0} \quad \text { at } t=0
$$

then this stays true for all later times $t$ in the comoving frame $\xi$. Only initial conditions that decay exponentially like $e^{-\lambda \xi}$ for $\xi \rightarrow \infty$, approach a solution with the larger velocity (18). Such an exponential decay is a very specific initial condition, furthermore, such a leading edge will generically be cut off for very small densities by the physical breakdown of the continuum approximation. Therefore the physically relevant solution is the one with velocity (19) and absent leading edge as in Eq. (20). The complete absence of the leading edge $(\lambda=\infty)$ is generic for the hyperbolic equation (2), i.e., for
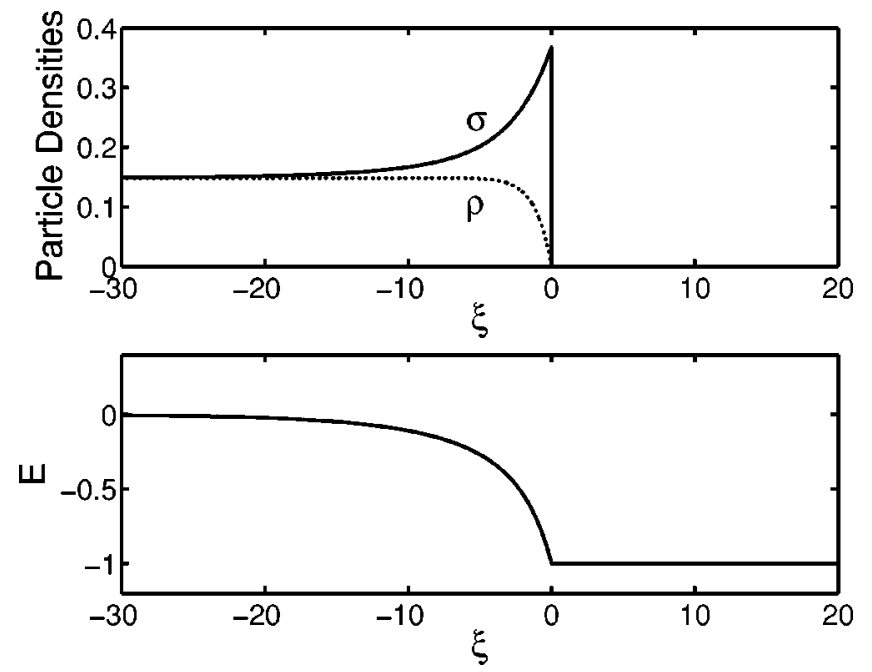

FIG. 1. Electron density $\sigma$ (solid line in first plot), ion density $\rho$ (dotted line in first plot), and electric field $E$ (second plot) for a negative ionization shock front moving with $v=\left|E_{\infty}\right|$ in the comoving frame $\xi=z-v t$. The far field is $E_{\infty}=-1$.

vanishing electron diffusion. We will restrict the analysis of fronts and their linear perturbations to propagation into a completely nonionized state (20) in the remainder of the paper.

In contrast to all other uniformly translating fronts with $v>-E_{\infty}$, the selected front with $v=-E_{\infty}$ exhibits a discontinuity of the electron density at some point $\xi$ which corresponds to $v+E(\xi) \rightarrow 0$. We choose the coordinates such that the discontinuity is located at $\xi=0$. The situation is shown in Fig. 1 for a uniformly translating front with velocity $v=1$ within a far field $E_{\infty}=-1$.

A discontinuity of $\sigma$ means that $\partial_{\xi} \sigma$ is singular at this position. On the other hand, the expression $\sigma(\rho-\sigma+f(E))$ in Eq. (10) is finite or vanishing, therefore the product $(v$ $+E) \partial_{\xi} \sigma$ in Eq. (10) may not diverge either. Hence $(v+E)$ has to vanish at the position of the discontinuity, and therefore $E=E_{\infty}=-v$ at the position of the front. Furthermore, since $(v+E) \rightarrow 0$ for $\xi \uparrow 0$, while $\partial_{\xi} \sigma$ is bounded for $\xi<0$ [as we will derive explicitly below in Eq. (28)] we have

$$
\lim _{\xi \rightarrow 0}[v+E(\xi)] \partial_{\xi} \sigma=0
$$

The fact that $\sigma(\xi)$ in Fig. 1 increases monotonically up to the position of the shock is generic and can be seen as follows: according to Eq. (10), and since $(v+E) \geqslant 0$ and $\sigma \geqslant 0$, the sign of $\partial_{\xi} \sigma$ is identical to the sign of $\sigma-\rho-f(E)$. With the help of the exact solutions (15) and (16), with the definition of $f(E)$ in Eq. (5) and with identifying $v=\left|E_{\infty}\right|$, we find

$$
\sigma-\rho-f(E)=|E| \int_{|E|}^{v} \frac{\alpha(x)-\alpha(E)}{v-|E|} d x \geqslant 0 .
$$

So $\sigma(\xi)$ increases monotonically for growing $\xi$ up to $\xi=0$ as long as $\alpha(E)$ increases monotonically with $E$. This is the 
case for Townsend form Eq. (6) or more generally for any $\alpha(E)$ that is monotonically increasing with $E$.

\section{Asymptotics near the shock front}

We now derive explicit expressions for $\sigma(\xi)$ etc. near the discontinuity. On approaching the position of the ionization shock front from below $\xi \uparrow 0$, the quantity

$$
\epsilon=v+E=\left|E_{\infty}\right|-|E|
$$

is a small parameter. The ion density Eq. (16) at this point can be expanded as

$$
\rho[E]=\rho[v-\epsilon]=\alpha(v) \epsilon-\alpha^{\prime}(v) \frac{\epsilon^{2}}{2}+O\left(\epsilon^{3}\right) .
$$

As the electron density is related to the ion density through $\sigma[E]=\rho[E] v / \epsilon$ according to Eq. (15), it is

$$
\sigma[E]=v \alpha(v)-v \alpha^{\prime}(v) \frac{\epsilon}{2}+O\left(\epsilon^{2}\right)
$$

Equation (17) evaluated for $E\left(\xi_{2}=0\right)=E_{\infty}<0$ reads

$$
-\xi=\int_{|E(\xi)|}^{v} \frac{v-x}{\rho[x]} \frac{d x}{x}=\int_{0}^{\epsilon} \frac{y}{\rho[v-y]} \frac{d y}{v-y},
$$

where in the last expression, the parameter $\epsilon$ (23) is introduced. Insertion of Eq. (24) now yields an explicit relation between $\xi$ and $E$,

$$
\begin{aligned}
-\xi & =\frac{\epsilon}{v \alpha(v)}+O\left(\epsilon^{2}\right) \\
\text { or } \quad \epsilon & =-v \alpha(v) \xi+O\left(\xi^{2}\right) .
\end{aligned}
$$

Insertion of this approximately linear relation between $\epsilon$ and $\xi$ into Eqs. (24) and (25) together with the notation $f(v)$ $=v \alpha(v)$ results in

$$
\begin{gathered}
\sigma(\xi)=\theta(-\xi)\left[f(v)+\frac{f(v) v \alpha^{\prime}(v)}{2} \xi+O\left(\xi^{2}\right)\right] \\
\rho(\xi)=\theta(-\xi)\left[-f(v) \alpha(v) \xi+O\left(\xi^{2}\right)\right] \\
-E(\xi)=v+\theta(-\xi)\left[f(v) \xi+O\left(\xi^{2}\right)\right]
\end{gathered}
$$

where we used $v=\left|E_{\infty}\right|$ and the step function $\theta(x)$, defined as $\theta(x)=1$ or 0 for $x>0$ or $x<0$, respectively.

\section{E. Asymptotics far behind the shock front}

Far behind the front in the ionized region $\xi \rightarrow-\infty$, the fields approach $\lim _{\xi \rightarrow-\infty}(\sigma, \rho, E)=\left(\sigma^{-}, \rho^{-}, E^{-}\right)$with

$$
\sigma^{-}=\rho^{-}=\int_{0}^{v} \alpha(x) d x, \quad E^{-}=0 .
$$

Expanding about this point as $\sigma(\xi)=\sigma^{-}+\sigma_{1}(\xi)$ etc., we derive in linear approximation

$$
\partial_{\dot{\xi}}\left(\begin{array}{c}
\sigma_{1} \\
\rho_{1} \\
-E_{1}
\end{array}\right)=\left(\begin{array}{ccc}
\lambda & -\lambda & 0 \\
0 & 0 & 0 \\
1 & -1 & 0
\end{array}\right)\left(\begin{array}{c}
\sigma_{1} \\
\rho_{1} \\
-E_{1}
\end{array}\right),
$$

with $\lambda$ given by

$$
\lambda=\frac{\sigma^{-}}{v}=\int_{0}^{v} \alpha(x) \frac{d x}{v} .
$$

Two eigenvalues of the matrix in Eq. (32) vanish. The third eigenvalue of the matrix is the positive parameter $\lambda$, it produces the eigendirection

$$
\left(\begin{array}{c}
\sigma \\
\rho \\
-E
\end{array}\right)(\xi)=\left(\begin{array}{c}
\sigma^{-} \\
\sigma^{-} \\
0
\end{array}\right)+A\left(\begin{array}{c}
\lambda \\
0 \\
1
\end{array}\right) e^{\lambda \xi}+O\left(e^{2 \lambda \xi}\right) \text { for } \xi \rightarrow-\infty
$$

which describes the asymptotic solution deep in the ionized region. The free parameter $A>0$ accounts for translation invariance.

\section{F. Two degeneracies of the shock front}

We have fixed the initial condition (20) and hence we have selected the front speed $v=-E_{\infty}$. Therefore the degeneracy of solutions related to the profile of the leading edge is removed. Still there are two degeneracies remaining in the problem. The first one is the well-known mode of infinitesimal translation that corresponds to the arbitrary position of the front. The second one is specific for the present problem and will play a central role in the derivation of the analytical asymptote for small $k$ in Sec. IV. It is the mode of infinitesimal change of far field $E_{\infty}$. It corresponds to the arbitrariness of the field $E_{\infty}$ in the nonionized region with $\sigma=0=\rho$ ahead of the front and to the corresponding arbitrariness of the asymptotic ionization level $\sigma=\sigma^{-}=\rho$ behind the front where the field vanishes. To set the stage for the later analysis, the necessary properties of the modes are given.

An infinitesimal translation of the front in space generates the linear mode $\left(\sigma_{t}, \rho_{t}, E_{t}\right)=\left(\partial_{\xi} \sigma, \partial_{\xi} \rho, \partial_{\xi} E\right)$,

$$
\begin{gathered}
(v+E) \partial_{\xi} \sigma_{t}=(2 \sigma-\rho-f) \sigma_{t}-\sigma \rho_{t}+\left(\sigma f^{\prime}-\partial_{\xi} \sigma\right) E_{t}, \\
v \partial_{\xi} \rho_{t}=-f \sigma_{t}+\sigma f^{\prime} E_{t}, \\
\partial_{\xi} E_{t}=\rho_{t}-\sigma_{t},
\end{gathered}
$$

with the definition $f^{\prime}=\partial_{x} f(|x|)$, so that $f\left(E+E_{t}\right)=f$ $-f^{\prime} E_{t}+\cdots$ for $E<0$. With the notation $\psi_{t}=-E_{t}$, the equations can be written in matrix form as

$$
\partial_{\xi}\left(\begin{array}{c}
\sigma_{t} \\
\rho_{t} \\
\psi_{t}
\end{array}\right)=\mathbf{N}_{0}(\xi) \cdot\left(\begin{array}{c}
\sigma_{t} \\
\rho_{t} \\
\psi_{t}
\end{array}\right)
$$




$$
\mathbf{N}_{0}(\xi)=\left(\begin{array}{ccc}
\frac{2 \sigma-f-\rho}{v+E} & \frac{-\sigma}{v+E} & \frac{\partial_{\xi} \sigma-\sigma f^{\prime}}{v+E} \\
\frac{-f}{v} & 0 & \frac{-\sigma f^{\prime}}{v} \\
1 & -1 & 0
\end{array}\right) .
$$

Note that the matrix $\mathbf{N}_{0}(\xi)$ reduces to the matrix in Eq. (32) for $\xi \rightarrow-\infty$, since $(2 \sigma-f-\rho) /(v+E) \rightarrow \sigma^{-} / v=\lambda$, etc. The limiting value of the vector $\left(\sigma_{t}, \rho_{t}, \psi_{t}\right)$ for $\xi \rightarrow 0$ is according to Eqs. (28)-(30),

$$
\left(\begin{array}{c}
\sigma_{t} \\
\rho_{t} \\
\psi_{t}
\end{array}\right) \stackrel{\xi \uparrow 0}{\rightarrow}\left(\begin{array}{c}
f v \alpha^{\prime} / 2 \\
-f \alpha \\
f
\end{array}\right)
$$

The second mode is generated by an infinitesimal change of the far field $E_{\infty}$ and consecutively by an infinitesimal change of the velocity $v$. The discontinuity is taken at the position $\xi=0$. In linear order, this variation creates a mode

$$
\sigma_{E}(\xi)=\lim _{\epsilon \rightarrow 0} \frac{\sigma^{\left[E_{\infty}+\epsilon\right]}(\xi)-\sigma^{\left[E_{\infty}\right]}(\xi)}{\epsilon} \text { etc. }
$$

which solves the inhomogeneous equation

$$
\partial_{\xi}\left(\begin{array}{c}
\sigma_{E} \\
\rho_{E} \\
\psi_{E}
\end{array}\right)=\mathbf{N}_{0}(\xi) \cdot\left(\begin{array}{c}
\sigma_{E} \\
\rho_{E} \\
\psi_{E}
\end{array}\right)-\left(\begin{array}{c}
\partial_{\xi} \sigma /(v+E) \\
\partial_{\xi} \rho / v \\
0
\end{array}\right) .
$$

The inhomogeneity vanishes at $\xi \rightarrow-\infty$. Hence like the front solution itself and like the infinitesimal translation mode, also this mode has the eigendirection $\left(\delta \sigma^{-}, \delta \sigma^{-}, 0\right)$ $+A(\lambda, 0,1) e^{\lambda \xi}+\cdots$ asymptotically for $\xi \rightarrow-\infty$. The value of $\delta \sigma^{-}$is given by $\delta \sigma^{-}=\partial \sigma^{-} / \partial\left|E_{\infty}\right|=\alpha\left(E_{\infty}\right)$ according to Eq. (31). For $\xi \uparrow 0$, the limiting values of the fields are

$$
\left(\begin{array}{c}
\sigma_{E} \\
\rho_{E} \\
\psi_{E}
\end{array}\right) \stackrel{\xi \uparrow 0}{\rightarrow}\left(\begin{array}{c}
f^{\prime} \\
0 \\
1
\end{array}\right)
$$

which is the derivative of Eqs. (28)-(30) with respect to $v$ $=\left|E_{\infty}\right|$ at $\xi=0$.

\section{SETUP OF LINEAR STABILITY ANALYSIS}

We now can proceed to study the stability of a planar ionization shock front. The front propagates into the $z$ direction. The perturbations have an arbitrary dependence on the transversal coordinates $x$ and $y$. Within linear perturbation theory, they can be decomposed into Fourier modes. Therefore we need the growth rate $s(k)$ of an arbitrary transversal Fourier mode to predict the evolution of an arbitrary perturbation. Because of isotropy within the transversal $(x, y)$ plane, we can restrict the analysis to Fourier modes in the $x$ direction, so we study linear perturbations $\propto e^{s t+i k x}$. (The notation anticipates the exponential temporal growth of such modes.)
In general, there can be a degeneracy of the dispersion relation $s(k)$ for various profiles of the leading edge just as it is found also for the uniformly translating solutions in Sec. II B. The constraint of a nonionized initial condition (20) again will remove this degeneracy and fix $s(k)$. In the present section, we will derive the equations and the boundary conditions for the Fourier modes. In Sec. IV, we will solve them numerically and derive the analytical asymptotes (1).

\section{A. Equation of motion}

The linear perturbation theory could be set up within the coordinate system $(x, \xi=z-v t)$ that moves with the unperturbed constant velocity $v=\left|E_{\infty}\right|$. This would, of course, lead to a set of equations that are linear in the perturbation.

However, when the perturbation of a planar front grows, the position of the actual discontinuity of the electron density will deviate from the position of the discontinuity of the unperturbed front. Within the coordinate system $(x, \xi)$, this would lead to finite deviations within infinitesimal spatial intervals instead of infinitesimal deviations within finite intervals. This conceptual difficulty can be avoided by formulating the perturbation theory within the coordinate system of the position of the perturbed shock front $(x, \zeta)$ with

$$
\zeta=\xi-\Delta_{k}, \quad \xi=z-v t, \quad \Delta_{k}=\delta e^{i k x+s t},
$$

where $z$ is the rest frame, $\xi$ is the frame moving with the planar front, and $\zeta=0$ marks the line of electron discontinuity of the actual front. Therefore we write the perturbation as

$$
\begin{gathered}
\sigma(x, \zeta, t)=\sigma_{0}(\zeta)+\sigma_{1}(\zeta) \Delta_{k}(x, t), \\
\rho(x, \zeta, t)=\rho_{0}(\zeta)+\rho_{1}(\zeta) \Delta_{k}(x, t), \\
\phi(x, \zeta, t)=\phi_{0}(\zeta)+\phi_{1}(\zeta) \Delta_{k}(x, t),
\end{gathered}
$$

where $\sigma_{0}, \rho_{0}$, and $\phi_{0}$ are the electron density, ion density, and electric potential of the planar ionization shock front from the preceding section. But these planar solutions here are shifted to the position of the perturbed front $\zeta$. Therefore they do not move with their proper velocity $v=-\partial_{t} \xi$, but with a slightly different velocity $-\partial_{t} \zeta=v-s \Delta_{k}$. The price to pay is that the equations of the perturbation analysis become inhomogeneous, actually in a similar way as in Ref. [18]. The gain is that the derivation of the boundary conditions at the shock front becomes more comprehensible, and that later in Sec. VB the identification of the analytical solution for small $k$ with the mode $\left(\sigma_{E}, \rho_{E}, \psi_{E}\right)$ from the preceding section becomes quite obvious.

Substitution of the expressions (43) into (9) gives to leading order in the small parameter $\delta$,

$$
\begin{gathered}
\left(v+E_{0}\right) \partial_{\zeta} \sigma_{1}=\left(s+2 \sigma_{0}-\rho_{0}-f\right) \sigma_{1}-\sigma_{0} \rho_{1} \\
+\left(\partial_{\zeta} \sigma_{0}-\sigma_{0} f^{\prime}\right) \partial_{\zeta} \phi_{1}-s \partial_{\zeta} \sigma_{0}, \\
v \partial_{\zeta} \rho_{1}=-f \sigma_{1}+s \rho_{1}-\sigma_{0} f^{\prime} \partial_{\zeta} \phi_{1}-s \partial_{\zeta} \rho_{0}, \\
\left(\partial_{\zeta}^{2}-k^{2}\right) \phi_{1}=\sigma_{1}-\rho_{1}+k^{2} E_{0} .
\end{gathered}
$$


Here $f=f\left(E_{0}\right), f^{\prime}=\left.\partial_{|E|} f(|E|)\right|_{E_{0}}$, and $E_{0}=-\partial_{\zeta} \phi_{0}(\zeta)$ is the electric field of the uniformly translating front. As explained above, these equations are not completely linear in $\left(\sigma_{1}, \rho_{1}, \phi_{1}\right)$, but contain the inhomogeneities $s \partial_{\zeta} \sigma_{0}, s \partial_{\zeta} \rho_{0}$, and $k^{2} E_{0}$.

To elucidate the structure of Eq. (44), we drop all indices 0 and introduce the matrix notation

$$
\begin{aligned}
\partial_{\zeta}\left(\begin{array}{c}
\sigma_{1} \\
\rho_{1} \\
\psi_{1} \\
\phi_{1}
\end{array}\right) & =\mathbf{M}_{s, k} \cdot\left(\begin{array}{c}
\sigma_{1} \\
\rho_{1} \\
\psi_{1} \\
\phi_{1}
\end{array}\right)-\left(\begin{array}{c}
s \partial_{\zeta} \sigma /(v+E) \\
s \partial_{\zeta} \rho / v \\
-E k^{2} \\
0
\end{array}\right), \\
\mathbf{M}_{s, k}(\zeta) & =\left(\begin{array}{cccc}
\frac{s+2 \sigma-f-\rho}{v+E} & \frac{-\sigma}{v+E} & \frac{\partial_{\zeta} \sigma-\sigma f^{\prime}}{v+E} & 0 \\
\frac{-f}{v} & \frac{s}{v} & \frac{-\sigma f^{\prime}}{v} & 0 \\
1 & -1 & 0 & k^{2} \\
0 & 0 & 1 & 0
\end{array}\right) .
\end{aligned}
$$

Here we introduced the auxiliary field

$$
\psi_{1}=\partial_{\zeta} \phi_{1}
$$

which corresponds to the perturbation $E_{1}$ of the electric field, but with reversed sign.

\section{B. Boundary conditions at the discontinuity}

Having obtained the perturbation equations, we are now in the position to derive the boundary conditions. First we consider the boundary conditions at $\zeta=0$ where we make explicit use of the initial condition (20). The boundary conditions arise from the boundedness of the electron density to the left of the shock front at $\zeta \uparrow 0$, and from the continuity of all other fields across the position $\zeta=0$ of the shock front.

As discussed in Sec. II D, for the uniformly propagating shock front, the quantity $(v+E) \partial_{\zeta} \sigma$ vanishes as $\zeta \uparrow 0$, since $(v+E)$ vanishes and $\partial_{\zeta} \sigma$ is bounded. Since this should hold both for the full solution as well as for the unperturbed solution, it also holds for the perturbation

$$
\lim _{\zeta \uparrow 0}[v+E(\zeta)] \partial_{\zeta} \sigma_{1}=0
$$

Furthermore

$$
[v+E(\zeta)] \partial_{\zeta} \sigma_{1} \equiv 0 \text { for } \zeta \geqslant 0
$$

This identity is trivial for $\zeta>0$, but nontrivial for $\zeta=0$. When the explicit expressions (28)-(30) are inserted into Eq. (44), we find

$$
\begin{aligned}
(v+E) \partial_{\zeta} \sigma_{1}= & {[s+f(v)] \sigma_{1}-f(v) \rho_{1}-f(v) f^{\prime}(v) \psi_{1} } \\
& +\left(\psi_{1}-s\right) \partial_{\zeta} \sigma+O(\zeta)
\end{aligned}
$$

First of all, $\partial_{\zeta} \sigma$ is singular at $\zeta=0$, since $\partial_{\zeta} \sigma \propto \partial_{\zeta} \theta(-\zeta)$ $=-\delta(\zeta)$. Therefore Eq. (49) requires that the coefficient of $\partial_{\zeta} \sigma$ must vanish

$$
\psi_{1}(0)=s
$$

which gives the first boundary condition. Second, applying now Eq. (48) yields the second boundary condition

$$
[s+f(v)] \sigma_{1}(0)-f(v) \rho_{1}(0)-f(v) f^{\prime}(v) \psi_{1}(0)=0 .
$$

Due to the discontinuity, actually two boundary conditions (51) and (52) result from Eqs. (48) and (49).

In a second step the continuity of the other fields across $\zeta=0$ is evaluated. The continuity of $\rho$ we get from Eq. (11) and the fact that $\sigma$ and $|\mathbf{E}|$ are bounded for all $\xi$. It immediately yields the third boundary condition

$$
\rho_{1}(0)=0
$$

just like for the unperturbed equation. Finally, for the boundary conditions on field and potential, it is helpful that there is an exact solution for the nonionized region at $\zeta>0$ for a boundary with the harmonic form (42). Since ahead of the front there are no particles $\sigma=0=\rho$, there are also no space charges, and for the potential, one has to solve $\nabla^{2} \phi=0$ with the limit $\mathbf{E}=-\nabla \phi \rightarrow E_{\infty} \hat{\zeta}=-v \hat{\zeta}$ as $\zeta \rightarrow \infty$. The general solution for $\zeta>0$ is

$$
\begin{aligned}
\phi & =v \xi+\delta c e^{-k \xi} e^{i k x+s t} \\
& =v \zeta+\delta\left(v+c e^{-k \zeta}\right) e^{i k x+s t}+O\left(\delta^{2}\right),
\end{aligned}
$$

with the yet undetermined integration constant $c$. Here we chose the gauge $\phi_{0}(\xi=0)=0$ for the unperturbed electric potential.

Now $\phi$ always is continuous, and $\mathbf{E}=-\nabla \phi$ is continuous, because the charge density $|\rho-\sigma|<\infty$ in Eq. (4) everywhere. The continuity of $\phi$ at $\zeta=0$ implies

$$
\phi_{1}(0)=v+c
$$

the continuity of $\partial_{x} \phi$ yields the same condition, and the continuity of $\partial_{\zeta} \phi$ implies

$$
\psi_{1}(0)=-c k
$$

The five boundary conditions (51) - (53) and (55) - (56) determine the value of the integration constant

$$
c=-\frac{s}{k}
$$

in Eq. (54) and the values of the four fields at $\zeta=0$,

$$
\left(\begin{array}{c}
\sigma_{1} \\
\rho_{1} \\
\psi_{1} \\
\phi_{1}
\end{array}\right) \rightarrow\left(\begin{array}{c}
f^{\prime}(v) s f(v) /(s+f(v)) \\
0 \\
s \\
(v k-s) / k
\end{array}\right)
$$


Hence the explicit solution in the nonionized region $\zeta>0$ is

$$
\begin{gathered}
\sigma(x, \zeta>0, t)=0=\rho(x, \zeta>0, t), \\
\phi(x, \zeta>0, t)=v \zeta+\delta \frac{v k-s e^{-k \zeta}}{k} e^{i k x+s t}+O\left(\delta^{2}\right) .
\end{gathered}
$$

\section{Solution strategy and limits for $\zeta \rightarrow-\infty$}

We aim to calculate the dispersion relation $s=s(k)$ for fixed $k$. For any $s$ and $k$, the solution at $\zeta>0$ is given explicitly by Eq. (59). This solution determines the value of the fields (58) at $\zeta=0$ as a unique function of $s$ and $k$. The expression (58) is the initial condition for the integration of Eq. (45) towards $\zeta \rightarrow-\infty$. The requirement that the solution approaches a physical limit at $\zeta \rightarrow-\infty$ has to determine $s$ as a function of $k$. According to a counting argument, this is indeed the case, as will be explained now.

First, the limiting values of the fields at $\zeta=-\infty$ are comparatively easy: the total charge vanishes, hence $\sigma_{1}$ and $\rho_{1}$ approach the same limiting value $\sigma_{1} \rightarrow \sigma_{1}^{-}$and $\rho_{1} \rightarrow \sigma_{1}^{-}$, and the electric field vanishes, hence $\psi_{1} \rightarrow 0$ and $\phi_{1} \rightarrow 0$. Here the limiting values at $\zeta \rightarrow-\infty$ again were denoted by the upper index ${ }^{-}$as in Eq. (34).

Second, the eigendirections are determined by linearizing the equations of motion (45) about this asymptotics. In a calculation similar to the one from Sec. II F, one derives for $\zeta \rightarrow-\infty$,

$$
\begin{gathered}
\left(\begin{array}{c}
\sigma_{1} \\
\rho_{1} \\
\psi_{1} \\
\phi_{1}
\end{array}\right) \underset{\zeta \rightarrow-\infty}{\approx}\left(\begin{array}{c}
\sigma_{1}^{-} \\
\sigma_{1}^{-} \\
0 \\
0
\end{array}\right)+a_{1} e^{\lambda_{1} \zeta}\left(\begin{array}{c}
\lambda_{1}^{2}-k^{2} \\
0 \\
\lambda_{1} \\
1
\end{array}\right)+a_{2} e^{\lambda_{2} \zeta}\left(\begin{array}{l}
1 \\
1 \\
0 \\
0
\end{array}\right) \\
+a_{3} e^{k \zeta}\left(\begin{array}{l}
0 \\
0 \\
k \\
1
\end{array}\right)+a_{4} e^{-k \zeta}\left(\begin{array}{c}
0 \\
0 \\
-k \\
1
\end{array}\right)
\end{gathered}
$$

with the free parameters $a_{1}, a_{2}, a_{3}, a_{4}$, and $\sigma_{1}^{-}$and the eigenvalues

$$
\lambda_{1}=\frac{\sigma^{-}+s}{v}=\lambda+\lambda_{2}, \quad \lambda_{2}=\frac{s}{v}
$$

and $\lambda$ from Eq. (33).

For positive $s$ and $k$, all eigenvalues $\lambda_{1}, \lambda_{2}$, and $k$ are positive except for the fourth one $-k$. Hence the first three eigendirections approach the appropriate limit for $\zeta \rightarrow-\infty$, while the fourth one does not. Therefore a solution can only be constructed for

$$
a_{4}=0 .
$$

This condition determines the dispersion relation $s=s(k)$ when a solution of Eqs. (45) and (58) is integrated towards $\zeta \rightarrow-\infty$.

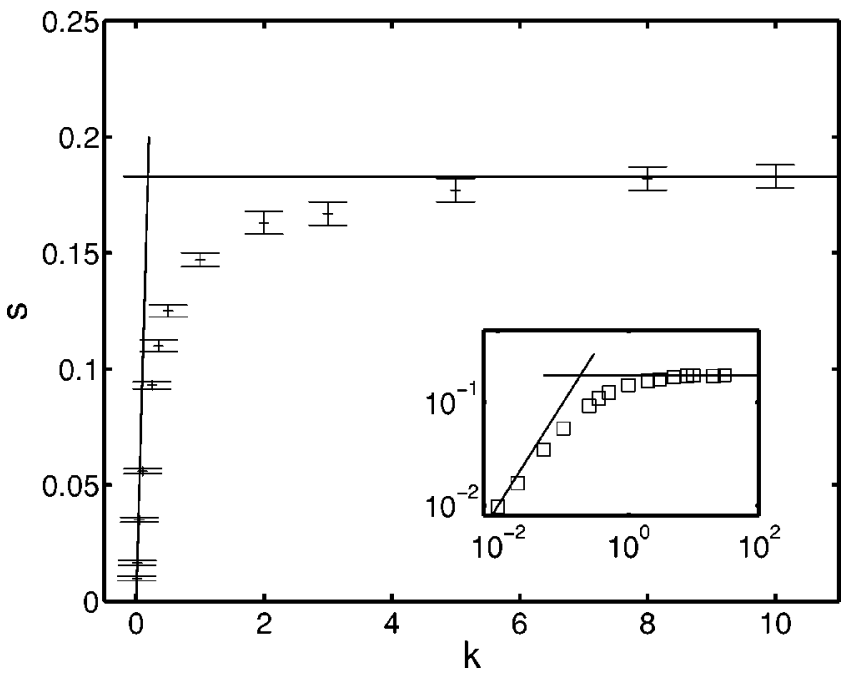

FIG. 2. Dispersion curve for $E_{\infty}=-1$, hence $v=1$. The big figure shows the numerical data with error bars and the two analytical asymptotes for small and large $k$ (lines). The inset shows the same data (squares) in double-logarithmic scale with the same two analytical asymptotes.

\section{CALCULATION OF THE DISPERSION RELATION}

Having set the stage, the dispersion relation is now first evaluated numerically for $E_{\infty}=-1$. Besides an expected result for small $k$, this investigation has delivered a previously unexpected result for large $k$. Based on these numerical results for fixed $E_{\infty}$, we were able to derive analytical asymptotes for small or large $k$ and for arbitrary $E_{\infty}<0$. We also understood the physical mechanism driving this asymptotic behavior. The section contains the derivation of our numerical results and of our analytical asymptotes and their physical interpretation.

\section{A. Numerical results for arbitrary $k$ and $\boldsymbol{E}_{\infty}=-1$}

The problem is to integrate the equations for the transversal perturbation (45) for fixed $k$ and guessed $s$ from the initial condition (58) at $\zeta=0$ towards decreasing $\zeta$. In general, the boundary condition (60) with Eq. (62) will not be met, so $s$ has to be iterated until $a_{4} \approx 0$. When the condition is met, the solution does not diverge for large negative $\zeta$, otherwise it does. When passing through the appropriate $s=s(k)$, the sign of the divergence changes. This is how the data points in Fig. 2 with their error bars were derived.

For the numerical integration, the ODEPACK collection of subroutines for solving initial value problems was used [22] to solve the seven ordinary differential equations for the unperturbed problem (10)-(12) and the perturbation (45)-(46) simultaneously. The unperturbed solution has to be calculated since it enters the matrix (46).

However, the numerics cannot directly be applied to the problem in the form (45)-(46) because the matrix contains apparently diverging terms proportional to $1 /[v+E(\zeta)]$ for $\zeta \rightarrow 0$. Therefore the behavior of the solution for $\zeta \rightarrow 0$ has to be evaluated in a similar way as in Sec. IIE. With the ansatz

$$
\sigma_{1}(\zeta)=\sigma_{1}\left(0^{-}\right)+C_{1} \zeta+O\left(\zeta^{2}\right)
$$




$$
\begin{aligned}
& \rho_{1}(\zeta)=\rho_{1}\left(0^{-}\right)+C_{2} \zeta+O\left(\zeta^{2}\right), \\
& \psi_{1}(\zeta)=\psi_{1}\left(0^{-}\right)+C_{3} \zeta+O\left(\zeta^{2}\right), \\
& \phi_{1}(\zeta)=\phi_{1}\left(0^{-}\right)+C_{4} \zeta+O\left(\zeta^{2}\right),
\end{aligned}
$$

where $\sigma_{1}\left(0^{-}\right)$, etc. are given by Eq. (58), the parameters $C_{i}$ become

$$
\begin{gathered}
C_{2}=-s \alpha\left(\frac{f f^{\prime}}{s+f}+f+f^{\prime}\right), \\
C_{3}=s\left(-k+\frac{f f^{\prime}}{s+f}\right), \quad C_{4}=s, \\
C_{1}=\frac{C_{2}+\left(\alpha+v \alpha^{\prime} / 2\right) C_{3}+s\left(v \alpha f^{\prime \prime}+v \alpha^{\prime} f^{\prime} / 2\right)}{2+s / f} .
\end{gathered}
$$

In the numerical procedure, the explicit solutions (28)-(30) and (63)-(64) are used until $\zeta=10^{-5}$, then the differential equations are evaluated.

The numerical results for the dispersion relation in a field $E_{\infty}=-1$, i.e., for a shock front with velocity $v=1$ are shown in Fig. 2. It can be seen that the dispersion curve for small $k$ grows linearly, but then turns over and finally for large $k$ saturates at a constant value.

\section{B. Asymptotics for small $k$ and arbitrary $\boldsymbol{E}_{\infty}<0$}

We first derive the asymptotic behavior for small $k$ for an arbitrary far field $E_{\infty}<0$.

When the equations of motion (45) and (46) are evaluated up to first order in $k, \phi_{1}$ decouples, and we get

$$
\partial_{\zeta}\left(\begin{array}{c}
\sigma_{1} \\
\rho_{1} \\
\psi_{1}
\end{array}\right)=\mathbf{N}_{s} \cdot\left(\begin{array}{c}
\sigma_{1} \\
\rho_{1} \\
\psi_{1}
\end{array}\right)-\left(\begin{array}{c}
s \partial_{\zeta} \sigma /(v+E) \\
s \partial_{\zeta} \rho / v \\
0
\end{array}\right)+O\left(k^{2}\right),
$$

where

$$
\mathbf{N}_{s}(\zeta)=\left(\begin{array}{ccc}
\frac{s+2 \sigma-f-\rho}{v+E} & \frac{-\sigma}{v+E} & \frac{\partial_{\zeta} \sigma-\sigma f^{\prime}}{v+E} \\
\frac{-f}{v} & \frac{s}{v} & \frac{-\sigma f^{\prime}}{v} \\
1 & -1 & 0
\end{array}\right)+O\left(k^{2}\right)
$$

is the truncated matrix $\mathbf{M}_{s, k}(\zeta)$ (46). The matrix $\mathbf{N}_{s}$ for $s$ $=0$ reduces to the matrix $\mathbf{N}_{0}$ from Eq. (37); this fact will be instrumental below. The fourth decoupled equation reads

$$
\partial_{\zeta} \phi_{1}=\psi_{1}
$$

The boundary condition $(58)$ reduces to

$$
\left(\begin{array}{c}
\sigma_{1} \\
\rho_{1} \\
\psi_{1}
\end{array}\right) \stackrel{\zeta \uparrow 0}{\longrightarrow}\left(\begin{array}{c}
f^{\prime} s f /(s+f) \\
0 \\
s
\end{array}\right)+O\left(k^{2}\right)
$$

and

$$
\phi_{1}(0)=\frac{v k-s}{k}
$$

Now compare the mode $\left(\sigma_{E}, \rho_{E}, \psi_{E}\right)$ of infinitesimal change of far field $E_{\infty}$ from Eqs. (39)-(41) to the present perturbation mode in the limit of small $k$. After identifying

$$
\left(\sigma_{1}, \rho_{1}, \psi_{1}\right)=\left(s \sigma_{E}, s \rho_{E}, s \psi_{E}\right),
$$

the equations and boundary conditions for the modes are identical in leading order of the small parameter $s$. Therefore the two modes have to become identical in the limit $s$ $\ll f(v)<v$. Integration over $\psi_{E}$ yields for the electric potential $\phi_{E}(0)-\phi_{E}(-\infty)=\int_{-\infty}^{0} d x \psi_{E}(x)$. This expression has to be of order unity since all other quantities are of order unity. But this implies that $\phi_{1}(0)$ due to Eq. (70) has to be of the order of $s$. Now compare the result for $\phi_{1}(0)$ in Eq. (69) which appears to depend in a singular way like $1 / k$ on the small parameter $k$. But for small $k$ and $s$ the expression $(v k$ $-s) / k$ indeed can be of the order of $s$, namely if

$$
s=v k+O\left(k^{3}\right) \quad \text { for } \quad k \ll \alpha(v) .
$$

This fixes the dispersion relation $s=s(k)$ in the limit of small $k$. The asymptote (71) is included as a solid line in Fig. 2.

\section{Physical interpretation of the small $k$ asymptote}

This result has an immediate physical interpretation: for small $k$, the wavelength of the transversal perturbation $2 \pi / k$ is the largest length scale of the problem. It is much larger than the thickness of the screening charge layer that is shown in Fig. 1. Therefore on the scale $1 / k$, the charged front layer is very thin and has the character of a surface charge rather than of a volume charge. This surface is equipotential according to Eq. (59) in linear approximation in the perturbation $\delta$, since

$$
\phi(x, \zeta=0, t)=\delta \frac{v k-s}{k} e^{i k x+s t}+O\left(\delta^{2}\right)=O(\delta k)+O\left(\delta^{2}\right)
$$

if we insert the dispersion relation $s=v k$ from Eq. (71). The corresponding electric field ahead of the interface is

$$
\mathbf{E}\left(x, \zeta=0^{+}, t\right)=-\left(v+\delta v k e^{i k x+s t}\right) \hat{\zeta}+O\left(\delta^{2}\right)
$$

in the same approximation. The small $k$ limit of the ionization front therefore is equivalent to an equipotential interface at position $\zeta=0$, i.e., at a position

$$
z(x, t)=v t+\delta e^{i k x+s t}
$$


in the rest frame $z$ (42). Its velocity in the $z$ direction is therefore

$$
v(x, t)=\partial_{t} z(x, t)=v+\delta v k e^{i k x+s t},
$$

where $s=v k$ was inserted. Comparison of Eqs. (73) and (75) shows that the interface moves precisely with the electron drift velocity $v=-E$ within the local field $E$.

We conclude that a linear perturbation of the ionization front whose wavelength is much larger than all other lengths, has the same evolution as an equipotential interface $(\phi$ $=$ const.) whose velocity is the local electron drift velocity $v=\nabla \phi$. It exhibits the familiar Laplacian interfacial instability $s \propto k$.

\section{Asymptotics for large $k$ and arbitrary $\boldsymbol{E}_{\infty}<0$}

For large wave vector $k$, the numerical results for the dispersion relation $s(k)$ in a field $E_{\infty}=-1$ approach a positive saturation value. We will now argue that the saturation value is given by $s(k)=f\left(E_{\infty}\right) / 2$. This asymptotic value, which for $\left|E_{\infty}\right|=1$ equals $e^{-1 / 2}=0.184$, is included as a solid asymptotic line in Fig. 2.

When the electron and ion densities remain bounded, the equations with the most rapid variation in Eqs. (45)-(46) for $k \gg 1$ are given by

$$
\begin{gathered}
\partial_{\zeta} \psi_{1}=k^{2} \phi_{1}+k^{2} E(\zeta)+O\left(k^{0}\right), \\
\partial_{\zeta} \phi_{1}=\psi_{1} .
\end{gathered}
$$

On the short length scale $2 \pi / k$, the unperturbed electric field for $\zeta<0$ can be approximated as in Eq. (30) by

$$
E(\zeta)=-v-f(v) \zeta+O\left(\zeta^{2}\right)
$$

so the equation for $\phi_{1}$ becomes

$$
\partial_{\zeta}^{2} \phi_{1}=k^{2}\left[\phi_{1}-v-f(v) \zeta\right] .
$$

The boundary condition (58) fixes $\phi_{1}(0)=(v k-s) / k$ and $\psi_{1}(0)=\partial_{\zeta} \phi_{1}=s$. The unique solution of Eq. (78) with these initial conditions is

$$
\phi_{1}(\zeta)=v+f(v) \zeta-\frac{f(v)}{2 k} e^{k \zeta}+\frac{f(v)-2 s}{2 k} e^{-k \zeta}
$$

for $\zeta<0$. Now the mode $e^{-k \zeta}$ would increase rapidly towards decreasing $\zeta$, create diverging electric fields in the ionized region and could not be balanced by any other terms in the equations. Therefore it has to be absent. The demand that its coefficient $[f(v)-2 s] / 2 k$ vanishes, fixes the dispersion relation

$$
s(k)=\frac{f(v)}{2}+O\left(\frac{1}{k}\right) \quad \text { for } \quad k \gg \alpha(v),
$$

which convincingly fits the numerical results for large $k$ in Fig. 2.

\section{E. Physical interpretation of the large $k$ asymptote}

Also for this result a physical interpretation can be given. First note that the $z$ component of the electric field on the discontinuity is

$$
E_{z}(x, \zeta=0, t)=-\left[v+\delta s e^{i k x+s t}+O\left(\delta^{2}\right)\right]
$$

with $s=f(v) / 2$. This is easily determined from either Eq. (59) or Eq. (43). Reasoning as in Eqs. (73)-(75), we again find that the shock line of the electron density moves with the local electron drift velocity-as it should.

Second, one needs to understand why the electric field on the shock line takes the particular form (81). In the frame $\xi$ $=z-v t$ of the unperturbed front (42), the electric field at the discontinuity is

$$
E_{z}(x, \xi=\Delta, t)=-\left(v+\frac{f(v)}{2} \Delta+O\left(\Delta^{2}\right)\right),
$$

where its position deviates with $\Delta(x, t)=\delta e^{i k x+s t}$ from the planar front.

In linear perturbation theory, the amplitude $\delta$ of the perturbation has to be much smaller than its wave length $2 \pi / k$. Since this wave length $2 \pi / k$ now is much smaller than the width of the front, the linear perturbation $\Delta$ explores only a small region around the position of the shock front. In this region, the electric field of the unperturbed front is according to Eq. (30) approximated by

$$
E_{z 0}(\xi)= \begin{cases}-\left[v+f(v) \xi+O\left(\xi^{2}\right)\right] & \text { for } \xi<0 . \\ -v & \text { for } \xi>0\end{cases}
$$

Therefore the electric field (82) is just the average over the behavior Eq. (83) for $\xi>0$ and $\xi<0$. This spatial averaging is enforced by the harmonic analysis of linear perturbation theory that will suppress different growth rates of positive or negative half-waves of the perturbation.

\section{F. A conjecture for the large $k$ asymptote}

We therefore conjecture: if the electric field of an unperturbed front is

$$
E_{0}(\xi)= \begin{cases}-\left(v+a \xi+O\left(\xi^{2}\right)\right) & \text { for } \xi<0 \\ -\left(v+b \xi+O\left(\xi^{2}\right)\right) & \text { for } \xi>0\end{cases}
$$

near the position of the discontinuity $\xi=0$, then a linear perturbation of this discontinuity with large $k$ will grow with rate

$$
s=\frac{a+b}{2} .
$$

If true, this behavior would have a stabilizing effect on large $k$ perturbations with growing curvature of the fronts, since the electric field decays in the nonionized region ahead of a curved front, therefore $b<0$. 


\section{CONCLUSIONS AND OUTLOOK}

We have studied the (in)stability of planar negative ionization fronts against linear perturbations. Such perturbations can be decomposed into transversal Fourier modes. We have determined the dispersion relation $s=s(k)$ shown in Fig. 2 numerically for a fixed field $E_{\infty}=-1$ far ahead of the front, and we have derived the analytical asymptotes

$$
s= \begin{cases}\left|E_{\infty}\right| k & \text { for } k \ll \alpha\left(\left|E_{\infty}\right|\right) / 2, \\ \left|E_{\infty}\right| \alpha\left(\left|E_{\infty}\right|\right) / 2 & \text { for } k \gg \alpha\left(\left|E_{\infty}\right|\right) / 2\end{cases}
$$

for arbitrary $E_{\infty}<0$. Since we have studied the minimal model, there is only one inherent length scale, namely the thickness of the charged layer as shown in Fig. 1. This thickness is approximated by $1 / \alpha\left(E_{\infty}\right)$. The wavelength $1 / k$ of the Fourier perturbation therefore has to be compared with this single intrinsic length scale $1 / \alpha\left(E_{\infty}\right)$ of the problem.

A specific property of our calculation is the expansion about a discontinuity of the electron density. Therefore we work in a coordinate system $\zeta=z-v t-\delta e^{i k x+s t}(42)$ that precisely follows the position of the discontinuity, and we explicitly distinguish in all calculations the nonionized region $\zeta>0$ from the ionized region $\zeta<0$. For the nonionized region $\zeta>0$, there is an exact analytical solution (59) for any $s$ and $k$ which determines the values of the fields at $\zeta=0$ as given in Eq. (58). Equation (58) serves as an initial condition for the integration towards $\zeta<0$. The approach towards $\zeta \rightarrow-\infty$ according to Eqs. (60) and (62) determines the growth rate $s$ as a function of $k$. In general, this calculation has to be performed numerically with results as shown in Fig. 2. The limits of small and large $k$ can be derived analytically. For small $k$, we can identify the perturbation mode with the mode of infinitesimal change of $E_{\infty}$. For large $k$, the growth rate corresponds to the evolution of the discontinuity in the unperturbed electric field averaged across the discontinuity. Both limits therefore have a simple physical interpretation.

The aim of the work was to identify a regularization for the interfacial model as suggested in Refs. $[8,11]$ and treated in Ref. [10]. Indeed, we have found that a Fourier mode for large $k$ in a far field $E_{\infty}=-v$ does not continue to increase with rate $s=v k$, but saturates at a value $s=f(v) / 2$. Still this is a positive value, and whether this suffices to regularize the moving boundary problem, remains an open question.

Besides this one, future work will have to investigate two more questions. First of all, there is the "simple" possibility to extend the model by diffusion. Diffusion is certainly going to suppress the growth rate of Fourier modes with large $k$ as our preliminary numerical work indicates. But there is also a second more subtle and interesting possibility: the growth rate of Fourier perturbations with large $k$ could change for a curved front, as we have conjectured in Sec. IV F. There we have argued that the saturating growth rate $s=f(v) / 2$ results from the average over the slope $-f(v)$ of the field in the ionized region and the slope 0 of the field in the nonionized region. For a curved front, the electric field in the nonionized region will have a slope of opposite sign that is proportional to the local curvature. We therefore expect the growth rate of a perturbation to decrease with growing curvature. These questions require future investigation.

\section{ACKNOWLEDGMENTS}

M. A. gratefully acknowledges hospitality of CWI Amsterdam and a grant from the EU-TMR-network "Patterns, Noise and Chaos."
[1] W.J. Yi and P.F. Williams, J. Phys. D 35, 205 (2002).

[2] E.M. van Veldhuizen and W.R. Rutgers, J. Phys. D 35, 2169 (2002).

[3] L. Niemeyer, L. Pietronero, and H.J. Wiesmann, Phys. Rev. Lett. 52, 1033 (1984).

[4] A.D.O. Bawagan, Chem. Phys. Lett. 281, 325 (1997).

[5] V.P. Pasko, U.S. Inan, and T.F. Bell, Geophys. Res. Lett. 28, 3821 (2001).

[6] H. Raether, Z. Phys. 112, 464 (1939).

[7] J.M. Meek and J.D. Craggs, Electrical Breakdown in Gases (Clarendon, Oxford, 1953).

[8] M. Arrayás, U. Ebert, and W. Hundsdorfer, Phys. Rev. Lett. 88, 174502 (2002).

[9] A. Rocco, U. Ebert, and W. Hundsdorfer, Phys. Rev. E 66, 035102 (2002).

[10] B. Meulenbroek, A. Rocco, and U. Ebert, Phys. Rev. E (to be published), e-print physics/0305112.

[11] E.D. Lozansky and O.B. Firsov, J. Phys. D 6, 976 (1973).

[12] U. Ebert, W. van Saarloos, and C. Caroli, Phys. Rev. Lett. 77, 4178 (1996); Phys. Rev. E 55, 1530 (1997).
[13] A.A. Kulikovsky, Phys. Rev. Lett. 89, 229401 (2002).

[14] U. Ebert and W. Hundsdorfer, Phys. Rev. Lett. 89, 229402 (2002).

[15] U. Ebert and M. Arrayás, in Coherent Structures in Complex Systems, edited by D. Reguera et al., Lecture Notes in Physics Vol. 567 (Springer Verlag, Berlin, 2001) pp. 270-282.

[16] S.K. Dhali and P.F. Williams, Phys. Rev. A 31, 1219 (1985); J. Appl. Phys. 62, 4696 (1987).

[17] P.A. Vitello, B.M. Penetrante, and J.N. Bardsley, Phys. Rev. E 49, 5574 (1994).

[18] U. Ebert and W. van Saarloos, Phys. Rev. Lett. 80, 1650 (1998); Physica D 146, 1 (2000).

[19] U. Ebert and W. van Saarloos, Phys. Rep. 337, 139 (2000).

[20] Y.P. Raizer, Gas Discharge Physics (Springer, Berlin, 1991).

[21] A.N. Lagarkov and I.M. Rutkevich, Ionization Waves in Electrical Breakdown in Gases (Springer, New York, 1994).

[22] A.C. Hindmarsh, in Scientific Computing, edited by R.S. Stepleman et al., IMACS Transactions on Scientific Computation Vol. 1 (North-Holland, Amsterdam, 1983), pp. 55-64. 